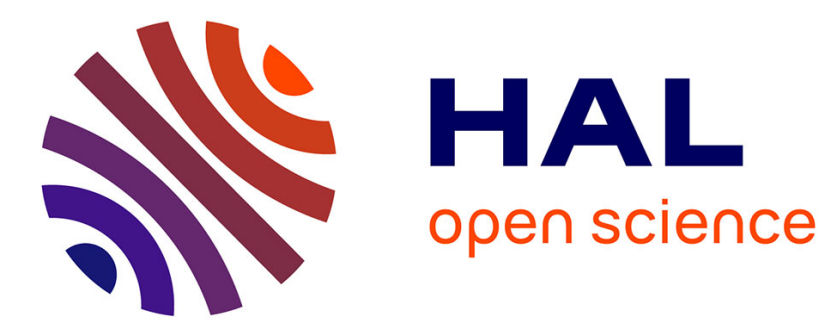

\title{
Le projet du Parc national du Mercantour
}

\author{
Louis Bergogne
}

\section{To cite this version:}

Louis Bergogne. Le projet du Parc national du Mercantour. Revue forestière française, 1971, 23 (S), pp.210-218. 10.4267/2042/20571 . hal-03394993

\section{HAL Id: hal-03394993 \\ https://hal.science/hal-03394993}

Submitted on 22 Oct 2021

HAL is a multi-disciplinary open access archive for the deposit and dissemination of scientific research documents, whether they are published or not. The documents may come from teaching and research institutions in France or abroad, or from public or private research centers.
L'archive ouverte pluridisciplinaire HAL, est destinée au dépôt et à la diffusion de documents scientifiques de niveau recherche, publiés ou non, émanant des établissements d'enseignement et de recherche français ou étrangers, des laboratoires publics ou privés. 


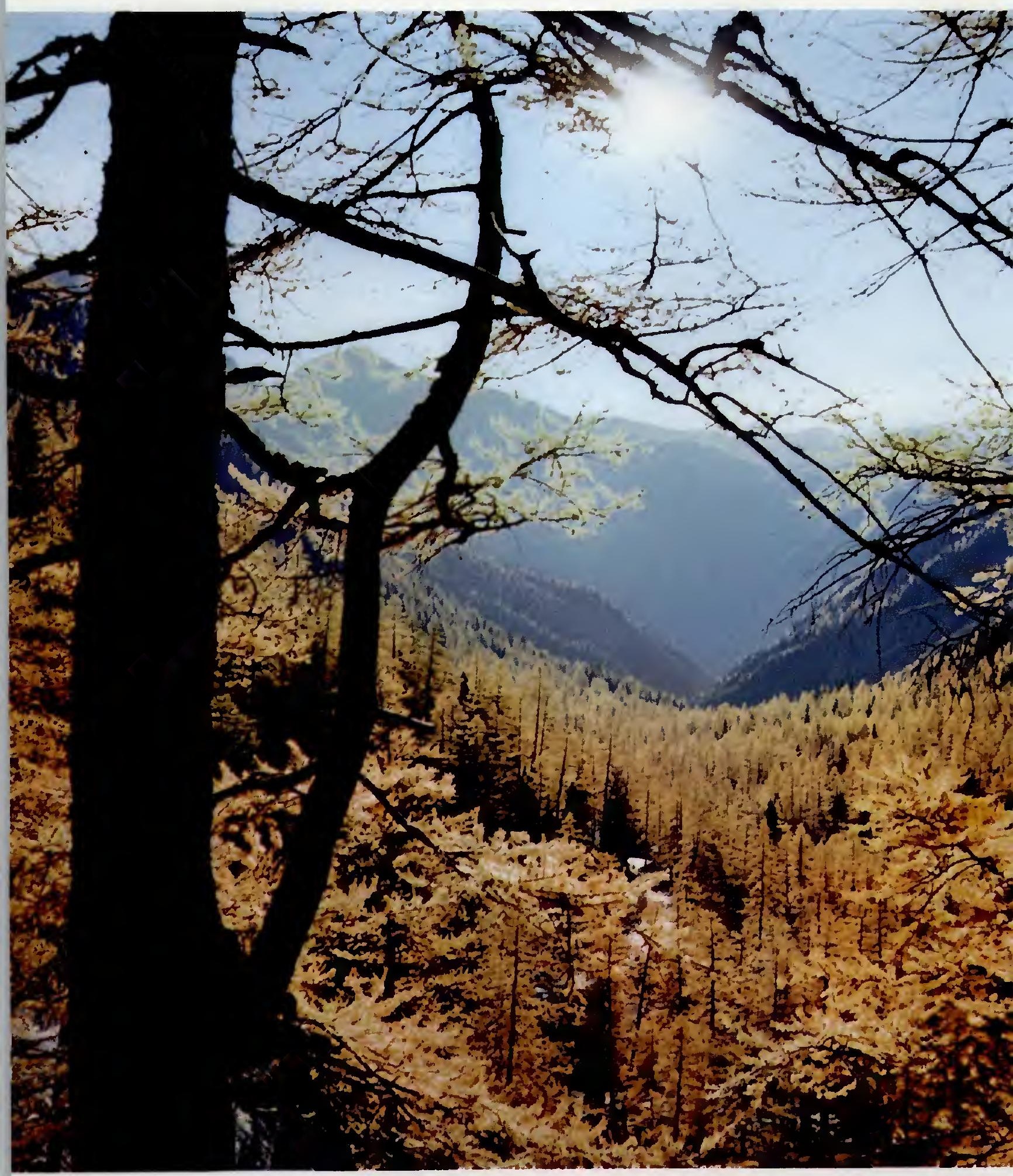

Photo SETTIMO 


\title{
LE PROJET DU PARC NATIONAL DU MERCANTOUR
}

\author{
L. BERGOGNE
}

Class. Oxford $(44 \times H-57 H) 907.11$

Le Mercantour est une puissante chaine de montagnes qul s'étend sur une soixantaine de kilomètres de part et d'autre de la frontiére franco-italienne, du col de Larche au col de Tende. Constitué principalement de gneiss, avec un noyau central de granite, il représente une résurgence de l'ossature cristalline des Alpes occidentales.

Sa longue crête crénelée se maintient toujours à une altitude élevée, dépassant généralement 2500 mètres et culminant à 3297 mètres à la polnte sud de l'Argentera en territoire italien.

Les principaux sommets se situent à moins de 80 kilomètres de la mer. II en résulte un relief brutal, tourmenté, avec des pentes extrêmement raides et généralement rocheuses.

Le versant nord, solidement épaulé par l'Argentera et le Mont-Matto (3088 m), tombe rapidement sur la plaine du Pô et forme une gigantesque muraille au sud-ouest de Coni.

Le versant français descend plus graduellement vers la Méditerranée. De l'arête centrale se détachent plusieurs crêtes secondaires qui poussent des ramifications dans tous les sens, formant selon l'expression de Raoul Blanchard, "le plus beau désordre qui soit de chaînes, de détilés et de gorges ".

Les routes contournent cette montagne masslve et dans la partie centrale, la plus compacte, seul le col de la Lombarde $(2351 \mathrm{~m})$ ouvre une brèche vers l'Italie.

L'ensemble est trés arrosé par les vents du golfe de Gênes et de nombreux torrents en descendent :

- du côté français, ce sont d'Est en Ouest: la Roya, la Vésubie, la Tinée et le Var,

- tandis que sur le flanc nord, le Vermenagna, les Gessos et la Stura di Demonte dessinent un superbe éventail en convergeant vers Coni.

Le versant méditerranéen a toujours relevé du Comté de Nice et aurait dú être rattaché en totalité à la France par le traité de Turín du 24 mars 1860.

Mais, par courtoisie pour le roi Victor Emmanuel II, et, sous le prétexte officiel de conserver à ce grand chasseur son domaine traditionnel de chasse, plus vraisemblablement du cóté italien pour des motifs d'ordre stratégique, la convention d'application du 17 mars 1861 traça une frontiére artificielle laissant sous la souveraineté italienne 30000 hectares en deçà de la ligne de partage des eaux.

Le traité du 10 février 1947 a mis fin à cette anomalie qui compliquait la gestion des territoires enclavés entre la frontière et la crête. 
Le classement des chasses royales et des terrains militaires en zones interdites a eu, du moins, l'heureuse conséquence d'assurer jusqu'à une date récente la protection des sites, de la faune et de la flore ainsi que la sauvegarde des richesses culturelles, d'une valeur exceptionnelle, que recèlent ces fonds de vallées.

Le Mercantour est une très belle montagne marquée par l'action des glaciers. Quelques-uns s'accrochent encore au flanc nord du Gelas $(3143 \mathrm{~m})$, le point culminant de l'actuelle frontière.

Toutes les vallées constituent de véritables musées de l'érosion glaciaire avec leur profil en forme d'auge, comme les vallées de Chastillon et de Mollières dans la Tinée, les vallons de Salèses, du Boréon, de Fenestre et de la Gordolasque dans la Vésubie et tout l'ensemble des Merveilles ou de la Valmasque dans la haute Roya, avec, à diftérents niveaux, une série de cirques et de cuvettes occupés par de nombreux lacs qui ne sont pas le moindre attrait du massif. On en compte 250 de part et d'autre de la frontière : lacs de Vens et de Rabuons (Tinée), lac Nègre (Mollières), lacs Bessons, lac de Très Colpas (Boréon), lac Long du Gelas, enfin, les multiples lacs des Merveilles et de la Valmasque.

On y découvre d'importants dépôts morainiques, des verrous, sciès en gorges ou franchis en cascades, ainsi que toute une série de roches moutonnées, de dalles usées, polies, cannelées ou striẻes par la glace, appelées ici des " ciappes ".

Le Mercantour offre aussi un large choix d'excursions, depuis les promenades faciles sur les pelouses herbeuses jusqu'aux escalades les plus sportives.

Très bien équipé en refuges par le Club alpin, il est devenu un grand centre d'alpinisme dont le Gélas et la Cougourde $(2912 \mathrm{~m})$, sont les cimes les plus prestigieuses.

Les spécialistes de la varappe s'accordent à vanter la qualité de ses courses ensoleillẻes, toujours jalonnées de fleurs éclatantes, ainsi que le panorama exceptionnel que l'on découvre du haut de ses sommets lorsque le regard, dominant la plaine du Pô, embrasse toute la courbe des Alpes vers le Pelvoux, Ie Mont-Blanc, le Cervin et jusqu'au Mont-Rose et porte, par delà la mer, jusqu'aux rivages de Corse.

C'est au mois d'août, bien sûr, qu'il y a foule dans le Mercantour mais c'est bien en automne, jusqu'au 15 novembre, que se situent les plus belles sorties quand les premiers froids ont teinté de pourpre les fustets ou les lentisques et que l'or des mélèzes chante dans les sombres vallées glaciaires.

Le climat de la Côte d'Azur a permis la culture d'un grand nombre d'arbres et de fleurs mais la flore autochtone est également d'une richesse inouïe puisque sur les 4500 espèces de l'herbier français on en trouve 2400 dans les Alpes-Maritimes.

On ne compte pas moins de 200 plantes rares dans le Comté de Nice, espèces originales qui n'existent qu'ici ou plantes d'autres pays qui ne dépassent pas la rive gauche du Var comme si la nature, ignorant le traité de Turin, ne reconnaissait que l'ancienne frontière.

Le Mercantour est le joyau botanique de cette région privilégiée à cause:

- de sa position géographique, entre les grandes Alpes et la Méditerranée, au contact de provinces aussi diverses que la Provence, la Ligurie, le Piémont et le Briançonnais, c'est-àdire au carrefour des trois domaines floristiques de la Méditerrannée, des Alpes et de l'Europe centrale ; variées:

- de son relief complexe et accidenté qui a créé des conditions ècologiques infiniment

- de son climat de haute montagne aux confins de la Méditerranée; le Grand Capelet (2 $934 \mathrm{~m}$ ) n'est qu'à 30 kilomètres de la mer, ce qui permet de curieux rapprochements, comme le mélèze près du chêne vert et l'olivier près du sapin ;

— des glaciations responsables de l'implantation sous cette latitude d'espèces boréales qui ont survécu en certains points ;

- de la variété des assises géologiques qui a favorisé l'éclosion de tous les éléments de la flore d'altitude, calcifuges sur les terrains siliceux du Mercantour et calcicoles dans le Marguareis dolomitique voisin.

La combinaison de ces différents facteurs a provoqué le rassemblement sur cette étroite bande, tout au long de la frontière, d'un nombre extraordinaire d'espèces d'origines variées et l'apparition de multiples formes locales. 


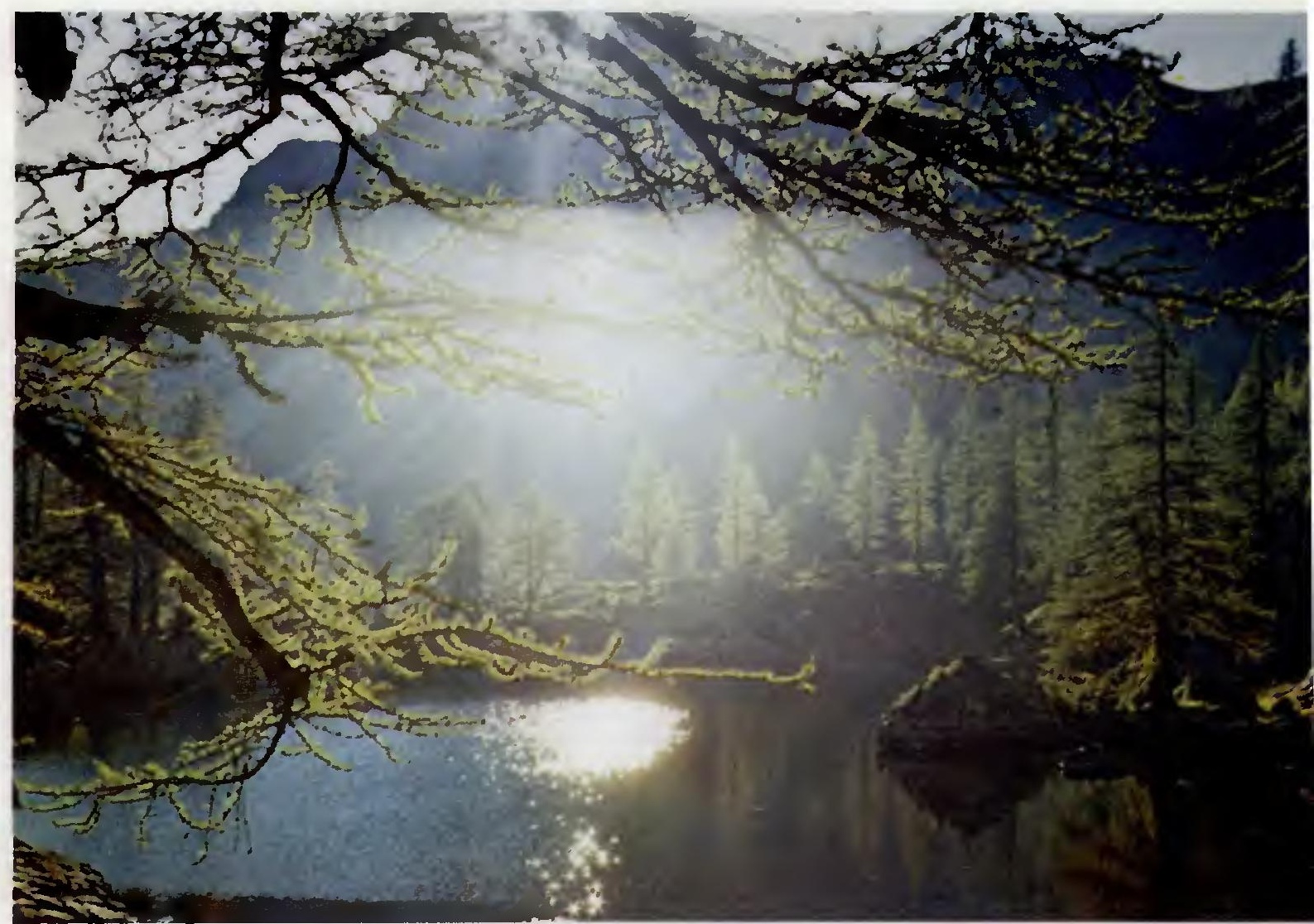

Vsillé des Mervellies

Photo SETTIMO

On y trouve notamment un lot important d'espèces d'Europe centrale ou orlentale, telles que le pin Mugho, et le Mercantour peut ainsi apparaître comme un morceau des Balkans accolé aux Alpes occidentales.

Mals c'est aussi la région d'Europe continentale la plus riche en espèces endémiques. Après un pointage sévère et négligeant les variétés, Ozenda en clte une vingtalne d'espéces dont la plus célébre est le magnifique Saxifraga florulenta qui décore de ses longues grappes roses, rarement épanouies, les falaises de la Gordolasque ou du Boréon, entre 2500 et 3000 mètres.

Ces conditions favorables se manifestent encore plus généreusement peut-être au profit de la faune et, notamment, des insectes pour lesquels le Mercantour est un refuge géographlque et paléontologique exceptionnel sinon un véritable creuset de biogénèse.

Les centres les plus riches se situent aux environs des cols: col de Tende, col de la Lombarde, col de la Cayolle.

En ce domaine, on note, comme pour la flore, de nombreux éléments endémiques, des formes d'Europe centrale ou orientale, des espèces boréo-alpines et des types ancestraux.

L'avifaune est également très intéressante avec le tétras lyre, le lagopède des Alpes, la perdrix bartavelle, deux ou trois couples d'aigle royal, deux couples de grand duc et la chouette de Tengmalm dont la présence dans le Mercantour a été signalée récemment. 


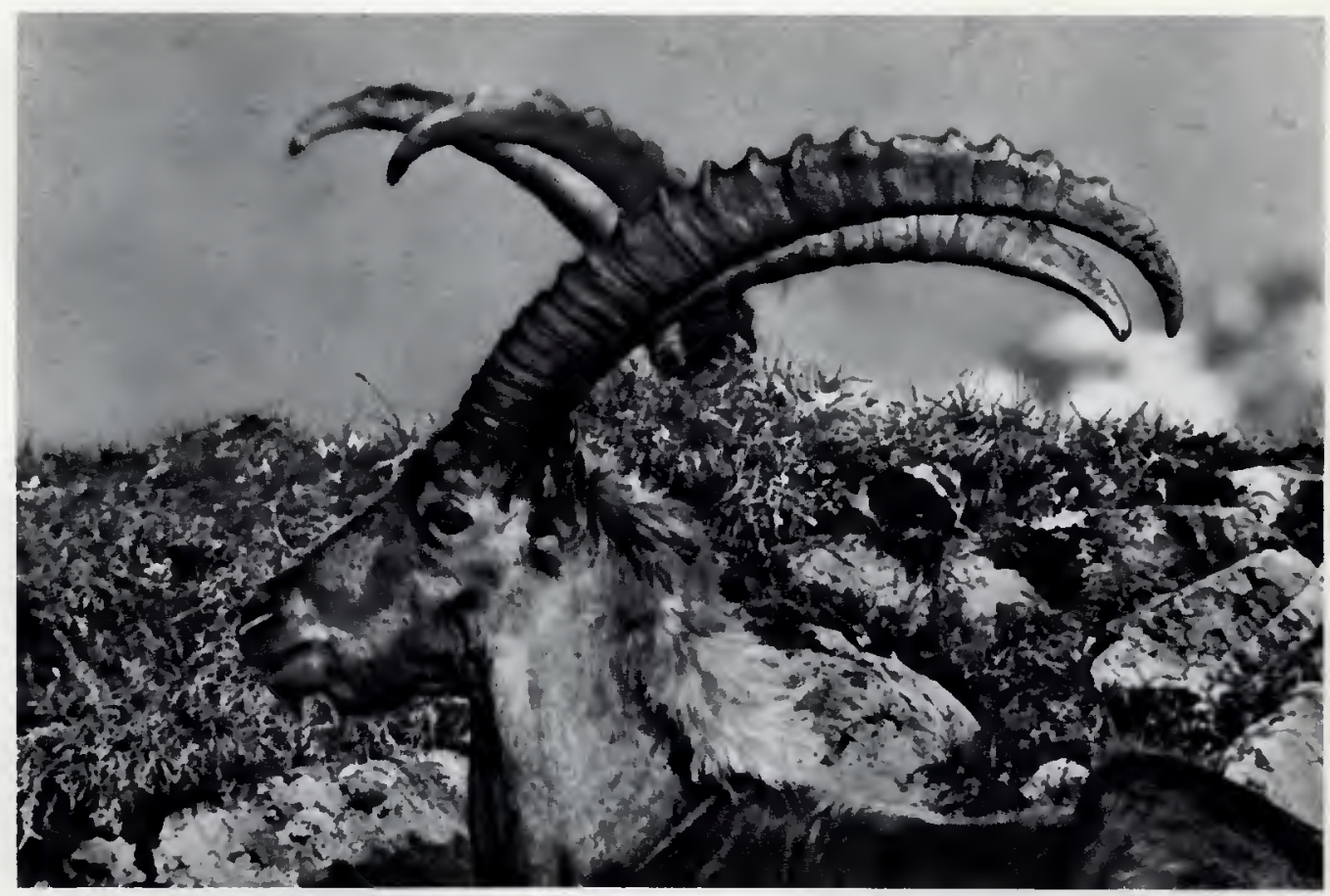

Bouquetins faisant le guet a la frontlère franco-italienne

Photo SETTIMO

Quant aux mammifères, ils ont très largement bénéficié de la mise en réserve d'intérêt national de 20000 hectares au centre du massif. On compte maintenant 3 à 4000 chamols, des bouquetins qui passent la frontière en été et des mouflons de Corse introduits avec succès il y a une dizaine d'années.

Le Mercantour, enfin, possède le plus riche ensemble de gravures rupestres à l'air libre existant actuellement en Europe.

Ces dessins primitifs, au nombre de 38 à 40000 , se sltuent dans un coin perdu, au fond d'une gorge étroite qui débouche dans la vallée de la Roya à Saint-Dalmas-de-Tende.

C'est un cirque d'une beauté sauvage drainé par les deux torrents de la Béonia et de la Valmasque qui font un tour complet autour du mont Bégo $(2873 \mathrm{~m})$, face au grand Capelet $(2935 \mathrm{~m})$ et à la cime du Diable $(2685 \mathrm{~m})$.

L'action des glaciers quaternaires y est peut-être plus saisissante que dans les vallées voisines. Ce ne sont que pentes abruptes, éboulis gigantesques et roches rabotées où se logent une trentaine de lacs.

C'est précisément sur les dalles de schiste dur, polies par l'érosion glaciaire ou " clappes" qu'ont été tracées par piquetage les gravures du mont Bégo.

On les découvre, entre 2000 et 2700 mètres, soit sur des blocs en place, soit sur des rochers éboulés, principalement dans la vallée dite des Merveilles et dans le val de Fontanalba.

Les sujets sont peu variés. Les plus nombreux sont des signes cornus, souvent réduits à un carré surmonté de deux cornes, droltes ou ondulées. On y voit aussl des enclos, avec le tracé des bâtiments et du bétail, des peaux de bêtes étalées au soleil, des instruments agricoles et des armes. Les figurations humaines sont rares.

Ces dessins dateralent de l'âge du bronze, du $3^{e}$ et du $2^{e}$ millénaire avant notre ére et pourraient avoir une signification religieuse. 
II s'agirait d'un culte topique, comme on en trouve maints exemples au cours de la préhistoire, voué au sommet des montagnes.

Le mont Bégo, cime prestigieuse, dominant toute la vallée de la Roya, toujours couronné de neige ou de nuages, était considéré comme une divinité, à la fois redoutable par le décor terrifiant qui s'étend à ses pieds et les orages qui éclatent si souvent à son front dans un fracas épouvantable, et bienfaisante par l'eau qu'il dispense généreusement pour abreuver le bétail et arroser les pâturages.

Les pasteurs primitifs qui, du Piémont ou de la côte, émigraient en été vers la montagne se rendaient au pied du Bégo déposer leurs oftrandes et graver sur ses flancs des ex-voto susceptibles d'attirer sur leurs troupeaux la protection divine.

Ces richesses archéologiques nous sont parvenues en assez bon état parce qu'elles étaient situées dans une zone de servitudes militaires et qu'elles n'étaient accessibles qu'à de rares bergers ou savants.

Aujourd'hui, la circulation est entièrement libre et la beauté du site attire chaque année davantage de visiteurs.

Les gravures sont maintenant très menacées et de nombreuses dégradations ont déjà été signalées : dessins passés à la craie pour les photographler, surchargés de graffiti stupides, grattés au couteau ou usés par le martelage des souliers à clous ou des cannes ferrées, et même, extraction de lamelles de schiste pour emporter un souvenir...

On ne saurait donc rester insensible à l'émouvant appel que lançait, à la veille du rattachement du Mercantour à la France, le Professeur Nino Lamboglia, directeur de l'Institut international d'études ligures à Bordighera:

"Nous contions à nos collègues trançais les gravures rupestres des Alpes-Maritimes en ce tournant de l'histoire qui les voit détachées de notre patrie.

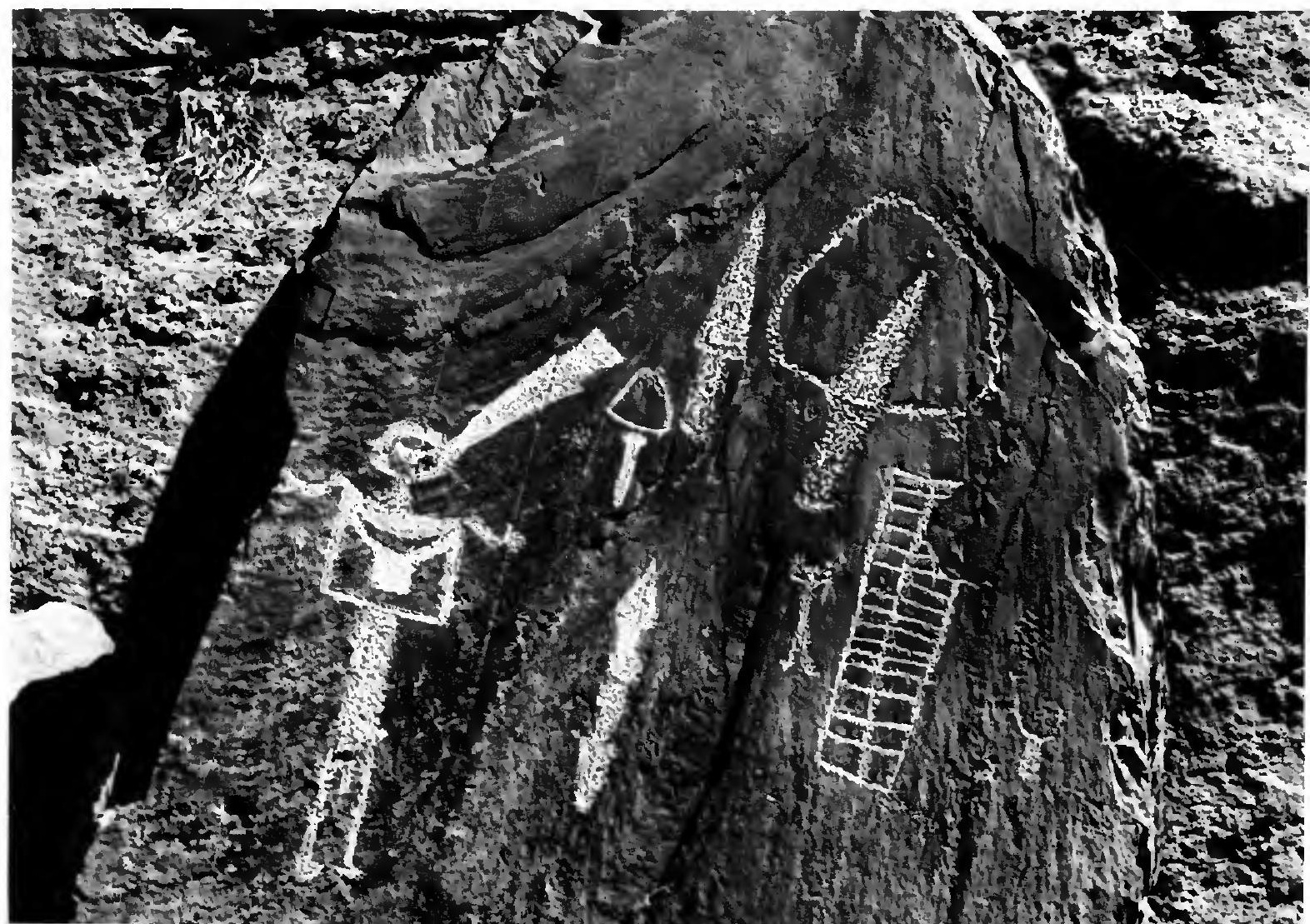


Nous le laisons à cœur ouvert et dans un esprit latin car nous sommes convaincus que tout comme aux origines, le génie ligure saura falre du mont Bégo un lieu de rendez-vous et un point de convergence intellectuelle entre l'Italie et la France. "

Le Mercantour apparaît ainsi comme la région de France la plus favorisée sous le rapport de la beauté des sites, de la richesse et de l'originalité de la flore et de la faune, de la valeur enfin des vestiges archéologiques.

Le versant italien est tout aussi remarquable et l'ensemble représente un domaine touristique et culturel de plus de 60000 hectares.

Ce territoire longtemps interdit et encore mal connu renferme un patrimoine naturel et humain unique en Europe et du plus grand intérêt pour la Science.

Mais Il est très menacé.

Plusieurs routes pénètrent maintenant au cœur du massit. La libre circulation des visiteurs de plus en plus nombreux met en péril les témoignages du passé. On déplore déjà la disparition ou le recul d'espéces animales ou végétales dont le Mercantour est le berceau ou le. dernier refuge. Des travaux ont altéré des sites inviolés jusqu'à ce jour et plusieurs projets de création de stations de sports d'hiver ou de résidences d'été ont été élaborés sans tenir compte de tous les intérêts en cause.

L'étude du projet de Parc national du Mercantour présente donc un réel caractère d'urgence pour assurer la sauvegarde des richesses exceptionnelles de cette région privllégiée.

Le Parc national permettrait aussi de ranimer l'économie en déclin constant des hautes vallées du Var, de la Tinée, de la Vésubie et de la Roya:

- par la renommée européenne et internationale que lui conféreraient son originalité, sa situation entre les Alpes et la Méditerranée, entre la Provence et le Piémont et son extension éventuelle sur le territoire italien :

- par le programme de travaux réalisés dans sa zone périphérique qui deviendrait un centre prioritaire de rénovation rurale, et qui engloberait les communes les plus défavorlsées du département des Alpes-Maritimes;

- par l'appoint aussi qu'il apporterait en été aux stations de sports d'hiver dont la rentabilité souffrira toujours de l'isolement géographique et restera soumise, quoiqu'on dise, aux caprices des saisons et aux fluctuations de l'économie nationale.

En vèrité, le Parc national du Mercantour serait un nouveau et splendide fleuron à inscrire au patrimolne touristique de la Côte d'Azur et des Alpes-Maritimes puisque, à deux heures de route de la mer et des plages, II offrirait une nature Infiniment riche et variée dans des montagnes reposantes et belles. 